\title{
Biosynthesis of Vitamin $\mathrm{B}_{12}$ : Stepwise Amidation of Carboxyl Groups $b, d, e$, and $g$ of Cobyrinic Acid $a, c$-Diamide Is Catalyzed by One Enzyme in Pseudomonas denitrificans
}

\author{
FRANCIS BLANCHE, ${ }^{1 *}$ MICHEL COUDER,${ }^{1}$ LAURENT DEBUSSCHE, ${ }^{1}$ DENIS THIBAUT, ${ }^{1}$ \\ BEATRICE CAMERON ${ }^{2}$ AND JOEL CROUZET ${ }^{2}$ \\ Département Analyse ${ }^{1}$ and Unité de Biologie Moléculaire, Institut des Biotechnologies, ${ }^{2}$ Centre de Recherche \\ de Vitry-Alfortville, Rhône-Poulenc Rorer S.A., B.P. 14, 94403 Vitry-sur-Seine Cedex, France
}

Received 11 March 1991/Accepted 25 July 1991

\begin{abstract}
The cobalamin biosynthetic pathway enzyme that catalyzes amidation of 5 '-deoxy-5' -adenosyl-cobyrinic acid $a, c$-diamide was purified to homogeneity from extracts of a recombinant strain of Pseudomonas denitrificans by a four-column procedure. The purified protein had an isoelectric point of 5.6 and molecular weights of 97,300 as estimated by gel filtration and 57,000 as estimated by gel electrophoresis under denaturing conditions, suggesting that the active enzyme is a homodimer. Stepwise Edman degradation provided the sequence of the first 16 amino acid residues at the $\mathbf{N}$ terminus. The enzyme catalyzed the four-step amidation sequence from cobyrinic acid a,c-diamide to cobyric acid via the formation of cobyrinic acid triamide, tetraamide, and pentaamide intermediates. The amidations are carried out in a specific order; this order was not determined. The enzyme was specific to coenzyme forms of substrates and did not carry out amidation of the carboxyl group at position $f$. The amidation reactions were $\mathrm{ATP} / \mathrm{Mg}^{2+}$ dependent and exhibited a broad optimum around $\mathrm{pH}$ 7.5. $\mathrm{L}$-Glutamine was shown to be the preferred amide group donor $\left(K_{m} \simeq 45 \mu \mathrm{M}\right)$ but could be replaced by ammonia $\left(K_{m}=20 \mathrm{mM}\right)$. For all of the four partially amidated substrates, the $K_{m}$ values were in the micromolar range and the $V_{\max }$ values were about $7,000 \mathrm{nmol} \mathrm{h} \mathrm{h}^{-1} \mathrm{mg}^{-1}$.
\end{abstract}

The biosynthesis of cobinamide from cobyrinic acid involves six stepwise amidations of peripheral carboxyl groups at positions $a, b, c, d, e$, and $g$ to yield free amides and attachment of $(R)$-1-amino-2-propanol at position $f$ (Fig. 1). In several laboratories, a number of partially amidated corrinoids have been isolated from cultures of microorganisms or transformed into complete corrinoids during feeding experiments to discover the sequence for attachment of unsubstituted amide groups and of $(R)$-1-amino-2-propanol (for a review, see reference 13). Some authors concluded that the amidations follow a preferred but not strictly obligatory route and that the $(R)$-1-amino-2-propanol group is introduced at a stage that depends on cultivation conditions $(2,13)$, whereas other authors suggested that $(R)$-1-amino-2propanol is incorporated only after completion of the six other amidations (1) and that the preceding amidations follow one unique sequence (Fig. 2) $(5,14,15,18)$.

The major corrinoids occurring in nature apparently exist in the coenzyme form (Fig. 1). The 5'-deoxy-5'-adenosyl (Ado) axial upper ligand of cobalt is presumably introduced soon after completion of the corrin ring, at a stage that is not established yet but certainly after the cobyrinic acid stage, since this intermediate has been isolated only in the diaqua form (2).

The first amidation activity along the cobalamin pathway from cobyrinic acid was recently purified to homogeneity from a recombinant strain of Pseudomonas denitrificans and was characterized in this laboratory (11). The enzyme was shown to catalyze the conversion of both cobyrinic acid and hydrogenobyrinic acid to their $a, c$-diamide derivatives via the transient formation of the $c$-monoamide. Our study also

\footnotetext{
* Corresponding author.
}

revealed that the two amidations of acetic carboxyls $c$ and $a$ of cobyrinic acid can precede adenosylation.

So far, only two in vitro studies dealing with amidation reactions of downstream cobyrinic acid $a, c$-diamide have been reported. The first involved incubations of Ado-corrinoid monocarboxylic to pentacarboxylic acids with crude cell extracts of Propionibacterium shermanii (20), and the second reports on an assay of a weak amidation activity with a synthetic substrate, presumably cobinic acid $a, c, d, e, g-$ pentaamide (structured like cobinamide, but with a free carboxyl group at position $b$; Fig. 1), with protein extracts of Clostridium tetanomorphum (12). These two studies, particularly that reported by Rapp (20), mainly confirmed the conclusions drawn from earlier in vivo work on the overall pathway (2) but did not provide information on the enzymology of the individual steps.

This work was undertaken to characterize the amidation activity from cobyrinic acid $a, c$-diamide. The enzyme was purified to homogeneity by high-performance liquid chromatography (HPLC) and was shown to catalyze the four stepwise amidations of carboxyl groups at positions $b, d, e$, and $g$ to yield cobyric acid.

\section{MATERIALS AND METHODS}

Bacterial strains and growth conditions. The bacteriological techniques used have been described elsewhere (6). Plasmid pXL617 (6) was transferred from Escherichia coli MC1060 (7) to $P$. denitrificans SC510 Rif ${ }^{\top}$ (4) through triparental mating with MC1060(pRK2073) as the source of the helper vector for conjugation $(6,19)$. For cobyric acid synthase purification, strain SC510 Rif'(pXL617) was grown on a rotary shaker at $30^{\circ} \mathrm{C}$ in $250-\mathrm{ml}$ Erlenmeyer flasks containing $50 \mathrm{ml}$ of PS4 medium (6) supplemented with 


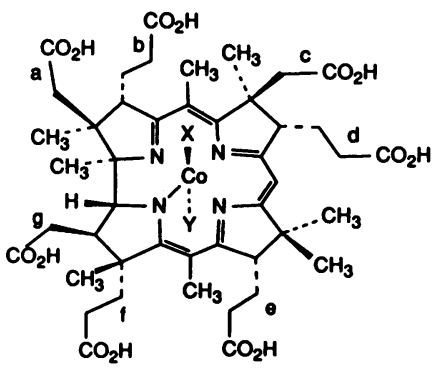

cobyrinic acid

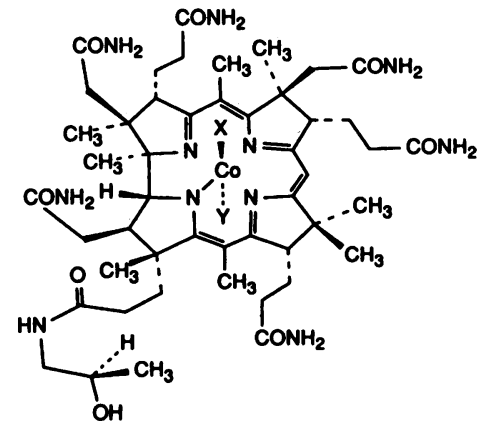

FIG. 1. Biosynthesis of cobinamide from cobyrinic acid. $X$ and $Y$ represent the axial upper and lower ligands of cobalt, respectively, and a through $\mathrm{g}$ represent the conventional lettering of peripheral side chains. For the coenzyme form $\mathrm{X}$ indicates $\mathrm{Ado}$ and $\mathrm{Y}$ indicates $\mathrm{H}_{2} \mathrm{O}$; for the diaqua form, both $\mathrm{X}$ and $\mathrm{Y}$ indicate $\mathrm{H}_{2} \mathrm{O}$.

lividomycin. After 4 days of incubation, the cells were harvested by centrifugation, washed with $0.1 \mathrm{M}$ Tris hydrochloride buffer ( $\mathrm{pH} 7.5)$, and stored at $-20^{\circ} \mathrm{C}$ until use.

Materials. Partially amidated corrinoids accumulated by growing $\boldsymbol{P}$. denitrificans SC510 Rif ${ }^{\top}$ cells were extracted and purified in their natural forms essentially as described elsewhere (5), except that cyanide was omitted throughout the purification process. These corrinoids were identified from their UV-visible spectra (17) and their chromatographic behaviors (5) after conversion to the corresponding cyano forms. The diaqua forms of cobyrinic acid $a, c$-diamide and cobyrinic acid pentaamide were generated from their coenzyme forms by sunlight photolysis as described by Weissbach et al. (22) and were identified from their UV-visible spectra (16). The monocyano forms of corrinoids and hydrogenobyrinic acid $a, c$-diamide were obtained as previously reported $(5,11)$. Centriprep 10 concentrators and Centricon 10 microconcentrators were obtained from Amicon Corp., Danvers, Mass.

General methods. The following methods used for protein characterization are described elsewhere $(4,11)$ : sodium dodecyl sulfate (SDS)-polyacrylamide gel electrophoresis, isoelectric focusing, estimation of protein molecular weight by gel filtration (on a Bio-Sil SEC 250 column; Bio-Rad), $\mathrm{N}$-terminal amino acid sequencing, and determination of protein concentrations.

Assay of cobyric acid synthase activity. The incubation conditions were those used previously for assaying cobyrinic acid $a, c$-diamide synthase activity (6), except that $1 \mathrm{mM}$ dithiothreitol (DTT) and $1 \mathrm{mM}$ EDTA were added to the incubation buffer ( $\mathrm{pH} 7.5$ ), and Ado-cobyrinic acid $a, c$ diamide or Ado-cobyrinic acid pentaamide $(10 \mu \mathrm{M})$ replaced hydrogenobyrinic acid. The amidated species formed during incubation were converted to their corresponding cyano forms and separated and quantitated by HPLC as described elsewhere (5). One unit of cobyric acid synthase activity was defined as the amount of enzyme necessary to transfer 1 nmol of amide group per $h$ under the conditions described above.

Kinetic measurements. The kinetic parameters were determined as previously described (11). All experiments were carried out at $\mathrm{MgCl}_{2}$ concentrations 2.5 -fold higher than the ATP concentrations. Appropriate equations were fitted to weighted experimental data with the computer program
Enzfitter (Elsevier Science Publishers BV, Amsterdam, The Netherlands).

Purification of cobyric acid synthase. Unless otherwise mentioned, the $\mathrm{pH}$ of all buffers was adjusted to 7.7 , and all chromatographic separations were carried out at $20^{\circ} \mathrm{C}$ with a Gilson 305 gradient HPLC system.

(i) Preparation of a crude extract. A cell extract of strain SC510 $\operatorname{Rif}^{\mathrm{T}}(\mathrm{pXL617})$ was prepared at $4^{\circ} \mathrm{C}$ from $2.5 \mathrm{~g}$ of wet cells in $10 \mathrm{ml}$ of $0.1 \mathrm{M}$ Tris hydrochloride-1 mM DTT-1 mM EDTA (buffer A) as previously described (4). Three volumes of $10 \mathrm{mM}$ Tris hydrochloride-1 mM DTT-20\% (wt/vol) glycerol (buffer B) were immediately added to the clear supernatant.

(ii) First ion-exchange chromatography. The crude cell extract was loaded at a flow rate of $3 \mathrm{ml} \mathrm{min}^{-1}$ on a Mono Q HR 10/10 column (Pharmacia, Uppsala, Sweden) equilibrated with buffer B. Proteins were eluted with a linear gradient of sodium chloride $(0$ to $0.5 \mathrm{M})$ in buffer $\mathrm{B}$.

(iii) Hydrophobic chromatography. One volume of buffer A-1.7 $\mathrm{M}$ ammonium sulfate was added to the active protein fraction from step ii, and the solution was loaded at a flow rate of $1.0 \mathrm{ml} \mathrm{min}{ }^{-1}$ onto a Phenyl-Superose HR 10/10 column (Pharmacia) equilibrated with buffer $\mathrm{A}-0.85 \mathrm{M}$ ammonium sulfate. Proteins were eluted with a linear decreasing gradient of ammonium sulfate from 0.85 to $0 \mathrm{M}$ in buffer A. Active fractions were pooled, and $20 \%$ glycerol was immediately added to the solution.

(iv) Second ion-exchange chromatography. The protein solution was concentrated to $2.5 \mathrm{ml}$ with a Centriprep 10 concentrator and desalted on a PD 10 column (Pharmacia) equilibrated with $50 \mathrm{mM}$ Tris hydrochloride (pH 8.3)-1 mM DTT-20\% glycerol (buffer C). The protein fraction was collected and injected at $1 \mathrm{ml} \mathrm{min}^{-1}$ on a Mono Q HR 5/5 column (Pharmacia) equilibrated with buffer C. Proteins were eluted with a linear gradient of sodium chloride (from 0 to $0.5 \mathrm{M}$ ) in buffer $\mathrm{C}$.

(v) Gel permeation chromatography. After concentration to $200 \mu \mathrm{l}$ with a Centricon 10 microconcentrator, the protein solution was fractionated on a Bio-Sil SEC-250 column (Bio-Rad Laboratories, Richmond, Calif.). The enzyme was eluted as a symmetrical peak at $0.5 \mathrm{ml} \mathrm{min}$ min $^{-1} 0 \mathrm{mM}$ Tris hydrochloride ( $\mathrm{pH} 7.5$ ) - $0.1 \mathrm{M}$ sodium chloride-1 mM DTT$20 \%$ glycerol. 

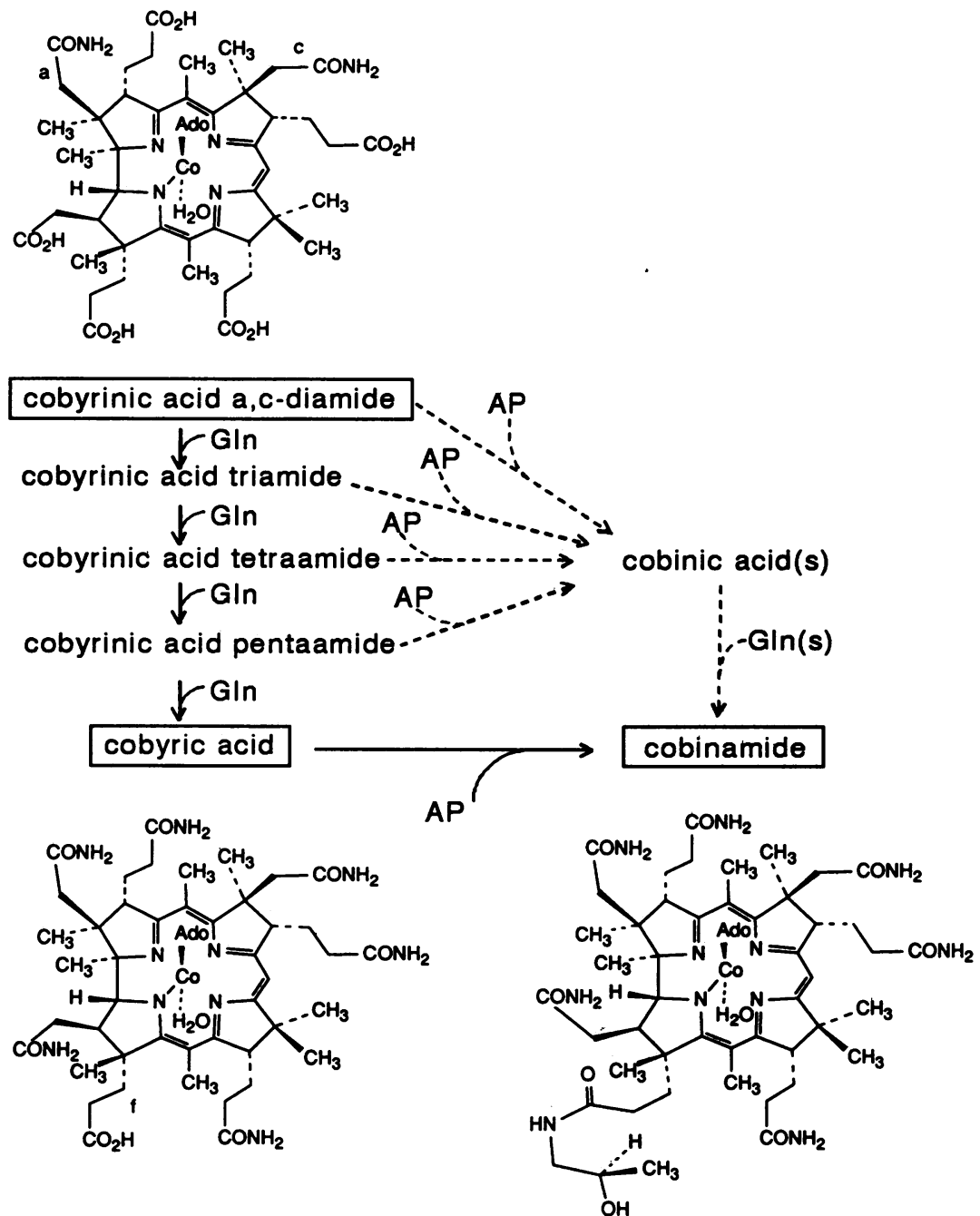

FIG. 2. Simplified scheme for biosynthetic reactions from cobyrinic acid $a, c$-diamide to cobinamide. The solid lines indicate the biosynthetic sequence demonstrated in $P$. denitrificans, and the dashed lines indicate alternate reaction sequences suggested in $P$. shermanii $(2,13)$. Gln, glutamine; AP, $(R)$-1-amino-2-propanol. The chemical structures for cobyrinic acid $a, c$-diamide, cobyric acid, and cobinamide are shown.

\section{RESULTS}

Identification of incompletely amidated corrinoids accumulated by $\boldsymbol{P}$. denitrificans. Earlier work in this laboratory (5) has shown that growing $\boldsymbol{P}$. denitrificans accumulates substantial amounts of cobyrinic acids $a, c$-diamide, triamide, tetraamide, and pentaamide as well as cobyric acid. However, despite careful examination, incompletely amidated corrinoids containing the $(R)$-1-amino-2-propanol group (cobinic acids) could not be detected in this microorganism. Therefore, it was suggested that, in $P$. denitrificans, the (R)-1-amino-2-propanol group is incorporated only after completion of all the other amidations (Fig. 2) (5). In this study, the partially amidated corrinoids were isolated and purified in their native forms. Only five compounds were found. All of them exhibited very similar spectra, with four maxima $(\varepsilon)$ at $262 \mathrm{~nm}\left(33,500 \mathrm{M}^{-1} \mathrm{~cm}^{-1}\right), 303 \mathrm{~nm}(21,000$ $\left.\mathrm{M}^{-1} \mathrm{~cm}^{-1}\right), 375 \mathrm{~nm}\left(8,500 \mathrm{M}^{-1} \mathrm{~cm}^{-1}\right)$, and $456 \mathrm{~nm}(9,000$ $\mathbf{M}^{-1} \mathrm{~cm}^{-1}$ ). These spectra, which remained almost unchanged after acidification, provided evidence that these compounds were incomplete $B_{12}$ factors containing an Ado group as the axial upper ligand of cobalt $(18,22)$. The compounds were further identified by HPLC as cobyrinic acids $a, c$-diamide, triamide, tetraamide, pentaamide, and cobyric acid after conversion to their corresponding cyano forms. The availability of Ado-cobyrinic acid $a, c$-diamide and the fact that this compound was the best candidate for being a substrate of the triamide synthase activity, prompted us to study the relevant enzymic step.

Detection of SC510 recombinant strains that amplify cobyric acid synthase activity and enzyme purification. An L-glutamine-dependent cobyrinic acid $a, c$-diamide amidating activity of about $100 \mathrm{U}$ mg of protein ${ }^{-1}$ was detected in crude extracts of SC510 Rif ${ }^{\mathrm{r}}$ strains harboring plasmid pXL617 or other plasmids carrying the $\operatorname{cob} Q$ gene (9). When they were separated by HPLC, the products of this activity were shown to be four in number. Three of them exhibited the same HPLC behavior as cobyrinic acids triamide, tetraamide, and pentaamide isolated from $P$. denitrificans, whereas the last one was cobyric acid. Amide synthase activity was undetectable $\left(<0.01 \mathrm{U} \mathrm{mg}^{-1}\right)$ in crude cell 
TABLE 1. Purification of cobyric acid synthase from $P$. denitrificans

\begin{tabular}{|c|c|c|c|c|c|c|}
\hline \multirow{2}{*}{$\begin{array}{l}\text { Purification } \\
\text { step }\end{array}$} & \multirow{2}{*}{$\begin{array}{l}\text { Vol } \\
(\mathrm{ml})\end{array}$} & \multirow{2}{*}{$\begin{array}{l}\text { Amt of } \\
\text { protein } \\
(\mathrm{mg})\end{array}$} & \multicolumn{2}{|c|}{$\begin{array}{l}\text { Sp act }\left(U^{m g}\right. \\
\text { of protein }\end{array}$} & \multirow{2}{*}{$\begin{array}{c}\text { Recovery }^{a} \\
(\%)\end{array}$} & \multirow{2}{*}{$\begin{array}{c}\text { Purifi- } \\
\text { cation }^{a} \\
\text { (fold) }\end{array}$} \\
\hline & & & $\mathrm{A}^{a}$ & $\mathbf{B}^{b}$ & & \\
\hline Crude extract & 8.5 & 203 & 110 & 120 & & \\
\hline Mono Q 10/10 & 8.0 & 35.5 & 390 & 420 & 62 & 3.5 \\
\hline Phenyl-Superose & 8.0 & 3.23 & 2,000 & 2,000 & 29 & 18 \\
\hline Mono Q 5/5 & 1.0 & 1.20 & 4,500 & 4,100 & 24 & 41 \\
\hline Bio-Sil SEC-250 & 0.75 & 0.88 & 5,000 & $\mathrm{ND}^{c}$ & 20 & 45 \\
\hline
\end{tabular}

${ }^{a}$ With Ado-cobyrinic acid $a, c$-diamide as the substrate.

$b$ With Ado-cobyrinic acid pentaamide as the substrate.

${ }^{c} \mathrm{ND}$, not determined.

extracts of SC510 Rifr. It was ATP/ $\mathrm{Mg}^{2+}$ dependent and exhibited a broad maximum around $\mathrm{pH} 7.5$ (7.2 to 8.0) in Tris hydrochloride buffer. The addition of $1 \mathrm{mM}$ EDTA in incubation buffer always resulted in a two- to threefold increase of activity. The enzyme was purified 45 -fold with an

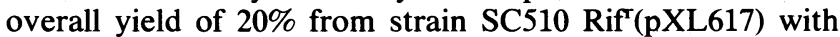
Ado-cobyrinic acid $a, c$-diamide as the substrate (Table 1). Glycerol $(20 \%, \mathrm{wt} / \mathrm{vol})$ and DTT $(\geq 1 \mathrm{mM})$ were necessary to stabilize the enzyme during purification $(100 \%$ of the activity was lost after $48 \mathrm{~h}$ at $0^{\circ} \mathrm{C}$ or $-20^{\circ} \mathrm{C}$ in $0.1 \mathrm{M}$ Tris hydrochloride [ $\mathrm{pH} 7.5])$. The enzyme could be stored at $-20^{\circ} \mathrm{C}$ for several weeks in the eluate from purification step $\mathrm{v}$ without detectable loss of activity.

Products of cobyric acid synthase activity. The products of purified cobyrinic acid $a, c$-diamide amidating activity were the same as those obtained by incubating impure protein fractions (i.e., cobyrinic acids triamide to pentaamide and cobyric acid); no fully amidated compound was detected (Fig. 3). Moreover, active fractions from all purification steps exhibited specific activities nearly identical when determined with cobyrinic acid $a, c$-diamide or cobyrinic acid

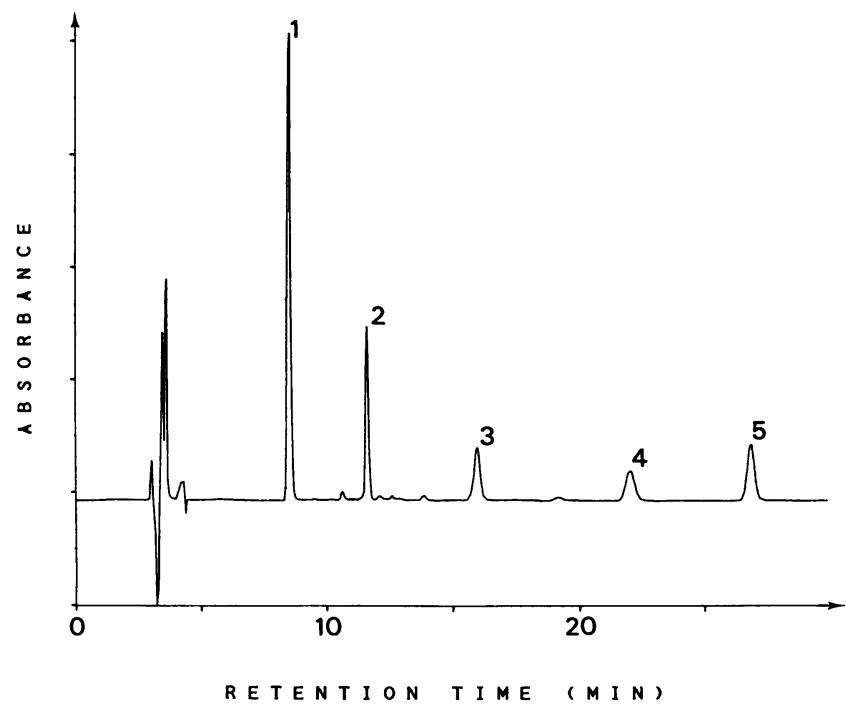

FIG. 3. Identification of products of purified cobyric acid synthase activity by HPLC. Peaks: 1 , cobyrinic acid $a, c$-diamide; 2 , cobyrinic acid triamide; 3 , cobyrinic acid tetraamide; 4 , cobyrinic acid pentaamide; 5 , cobyric acid. The incubation conditions were as described in Materials and Methods with Ado-cobyrinic acid $a, c$ diamide as a substrate.

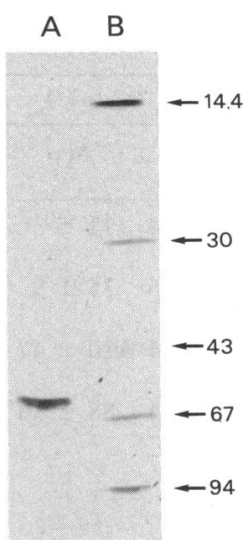

FIG. 4. SDS-polyacrylamide gel electrophoresis of purified cobyric acid synthase. Lanes: A, pure cobyric acid synthase $(0.5 \mu \mathrm{g})$; $\mathrm{B}$, molecular weight markers (in thousands) $(0.25$ to $0.5 \mu \mathrm{g}$ per band).

pentaamide as the substrate (within experimental error) (Table 1), thus indicating that the same enzyme is responsible for all four amidation reactions.

Homogeneity and N-terminal amino acid sequence. The purified protein was homogeneous, as judged by SDS-polyacrylamide gel electrophoresis (Fig. 4) and isoelectric focusing (isoelectric point, 5.6; data not shown). When the enzyme was subjected to microsequencing, the $\mathrm{N}$ terminus was not blocked and the first 16 cycles revealed only one clean sequence: $\mathrm{H}_{2} \mathrm{~N}$-Thr-Arg-Arg-Ile-Met-Leu-Gln-Gly-Thr-GlySer-Asp-Val-Gly-Lys-Ser.

Molecular weight. The molecular weight of the native enzyme was estimated to be $97,300 \pm 2,000$ by gel filtration, whereas SDS-polyacrylamide gel electrophoresis (Fig. 4) showed a molecular weight of $57,000 \pm 3,000$. These results strongly suggest a homodimeric structure.

Absorption spectrum. In $50 \mathrm{mM}$ Tris hydrochloride $(\mathrm{pH}$ 7.5)-5 mM DTT-1 mM EDTA-20\% glycerol, purified cobyric acid synthase had a typical protein spectrum with an absorption maximum at $280 \mathrm{~nm}$ and no absorbance in the visible region. The $A_{280} / A_{260}$ ratio was $1.86: 1$, and the measured molar extinction coefficient at $280 \mathrm{~nm}$ was $5.810^{4}$ $\mathrm{M}^{-1} \mathrm{~cm}^{-1}$.

Catalytic properties and substrate specificity. Steady-state kinetic analyses of the purified enzyme were carried out with Ado-cobyrinic acids $a, c$-diamide, triamide, tetraamide, and pentaamide. All of the four corrinoid substrates showed identical $K_{m}$ and $V_{\max }$ values (about $1.2 \mu \mathrm{M}$ and 7,000 nmol $\mathrm{h}^{-1} \mathrm{mg}^{-1}$, respectively; Table 2). Glutamine affinity for cobyric acid synthase was roughly constant regardless of the corrinoid substrate used $\left(K_{m}\right.$ value around $\left.45 \mu \mathrm{M}\right)$, whereas ATP affinity was apparently dependent on the number of free carboxyl groups present on peripheral side chains of the corrinoid used for the kinetic analysis. This phenomenon, which has potentially regulatory significance, was not investigated further. The positions of the amide groups in cobyrinic acids triamide, tetraamide, and pentaamide were not determined, and the actual sequence of the four amidations from cobyrinic acid $a, c$-diamide is not yet established. However, in the presence of an excess of cobyric acid synthase, the triamide, tetraamide, and pentaamide derivatives of cobyrinic acid transiently formed in the course of incubation were shown to be quantitatively transformed into cobyric acid at the end. This result indicates that (i) coby- 
TABLE 2. Steady-state parameters for turnover of some substrates by cobyric acid synthase

\begin{tabular}{|c|c|c|c|c|}
\hline \multirow[b]{2}{*}{ Substrate } & \multicolumn{3}{|c|}{$K_{m}(\mu \mathrm{M})$} & \multirow{2}{*}{$\begin{array}{c}\underset{\max }{V_{\max }} \\
\left(\mathrm{nmol}^{-1}\right. \\
\left.\mathrm{mg}^{-1}\right)\end{array}$} \\
\hline & Corrinoid & ATP & $\begin{array}{c}\text { Glu- } \\
\text { tamine }\end{array}$ & \\
\hline $\begin{array}{l}\text { Ado-cobyrinic acid } \\
a, c \text {-diamide }\end{array}$ & $1.04 \pm 0.24$ & $11 \pm 3$ & $45 \pm 11$ & 6,850 \\
\hline $\begin{array}{l}\text { Ado-cobyrinic acid } \\
\text { triamide }\end{array}$ & $1.22 \pm 0.26$ & $35 \pm 9$ & $33 \pm 7$ & 6,560 \\
\hline $\begin{array}{l}\text { Ado-cobyrinic acid } \\
\text { tetraamide }\end{array}$ & $1.21 \pm 0.04$ & $120 \pm 47$ & $64 \pm 9$ & 6,920 \\
\hline $\begin{array}{l}\text { Ado-cobyrinic acid } \\
\text { pentaamide }\end{array}$ & $1.25 \pm 0.24$ & $88 \pm 18$ & $49 \pm 5$ & 7,280 \\
\hline Ado-cobyric acid & & & & No amidation \\
\hline $\begin{array}{l}\text { Hydrogenobyrinic } \\
\text { acid } a, c \text {-diamide }\end{array}$ & & & & No amidation \\
\hline
\end{tabular}

rinic acids triamide to pentaamide are on the pathway to cobyric acid and (ii) the carboxyl group at position $f$ is not amidated by the enzyme. This later result was rigorously confirmed by incubating pure cobyric acid synthase with Ado-cobyric acid (Table 2).

This activity was apparently specific to the coenzyme form of the corrinoid substrate, since neither the monocyano nor diaqua form of cobyrinic acid $a, c$-diamide or cobyrinic acid pentaamide was amidated by cobyric acid synthase. Hydrogenobyrinic acid $a, c$-diamide was also not a substrate of the reaction (Table 2 ). The preferred amino group source was L-glutamine, but ammonia could be used instead with a reduced affinity $\left(K_{m}\right.$ of $20.4 \mathrm{mM}$ with cobyrinic acid $a, c$ diamide as a substrate).

\section{DISCUSSION}

The enzyme that catalyzes amidation of cobyrinic acid $a, c$-diamide was purified to homogeneity from extracts of a recombinant $\boldsymbol{P}$. denitrificans strain by a four-column procedure. The purified preparations were homogeneous, as judged from SDS-polyacrylamide gel electrophoresis, isoelectric focusing, and gel permeation. Moreover, when subjected to amino-terminal analysis, our final preparation showed only one sequence for the first 16 amino acid residues at the $\mathrm{N}$ terminus, which indicated complete removal of the terminal methionine in the mature protein. This sequence was identical with the $\mathrm{N}$-terminal sequence of the protein encoded by the $\operatorname{cob} Q$ gene carried by plasmid pXL617 (9), and the estimated molecular weight of the denatured protein $(57,000)$ agreed reasonably well with the molecular weight $(52,000)$ predicted from the DNA sequence of the $\operatorname{cob} Q$ gene. These results establish that $\operatorname{cob} Q$ is the structural gene of cobyric acid synthase. Evidence that cobyric acid synthase is involved in cobalamin biosynthesis was obtained from a study of strains G624 and G626, two Agrobacterium tumefaciens mutants carrying a cobQ mutation (6) complemented by pXL617. When cultivated in a ${ }^{57} \mathrm{Co}$-containing medium, these mutants where shown by HPLC (5) to be deficient in cobalamin biosynthesis $(<0.5 \%$ of the parent strain level), but they accumulated significant quantities of cobyrinic acid a,c-diamide (13.6 and 3.5\%, respectively, of the amount of cobalamin produced by the parent strain). No other cobalamin precursor was detected.

The purified protein appeared to catalyze the four-step amidation sequence of reactions from cobyrinic acid $a, c$ diamide to cobyric acid, acting on coenzyme forms of substrates only and using glutamine as the amide donor. Therefore the enzyme is a glutamine amidotransferase and must be classified as $5^{\prime}$-deoxy-5'-adenosylcobyrinic acid $a, c$-diamide:L-glutamine amido-ligase (or, more simply, Ado-cobyric acid synthase [glutamine hydrolyzing]) (21). The enzyme exhibited several properties common to glutamine amidotransferases (23). It was sensitive to oxydation, and the glutamine amide transfer activity was lost if a reducing agent was not included in all buffers. Moreover, the enzyme could utilize ammonia in place of glutamine.

The positions of amide groups in triamide, tetraamide, and pentaamide derivatives of cobyrinic acid were not determined. However, at each amidation stage the compound obtained was homogeneous when analyzed by HPLC (Fig. 3). Previous work in this laboratory has demonstrated the very high resolving power of this HPLC system. For instance, all of the monoacetamide and diacetamide derivatives of cobyrinic acid are completely separated in this system, and retention times of cobyrinic acid $a, c, g$-triamide and cobyrinic acid triamide isolated from $P$. denitrificans have been shown to be quite different (5). Therefore, the remote possibility that cobyrinic acids triamide, tetraamide, and pentaamide from $\boldsymbol{P}$. denitrificans are mixtures of isomers that are not separated at all in our HPLC system is very unlikely. This conclusion strongly suggests that the amidations from cobyrinic acid $a, c$-diamide to cobyric acid follow only one reaction sequence in a specific order. However, this order was not determined. This study also established that $g$ is not the first carboxyl group to be amidated by cobyric acid synthase and strengthened the view that the $(R)$-1-amino-2-propanol group is incorporated only after completion of all of the other amidations.

One may argue however, that the pattern of corrinoid accumulation in $\boldsymbol{P}$. denitrificans could be interpreted in the opposite manner. Let us assume, as illustrated in Fig. 2, that cobyrinic acid $a, c$-diamide is the natural substrate for the aminopropanol-inserting activity and that the resulting cobinic acid $a, c$-diamide is amidated into cobinamide by the product of the $\operatorname{cob} Q$ gene far more efficiently than the corresponding aminopropanol-free corrinoid. In this instance, the absence of accumulation of cobinic acid derivatives would indicate that the insertion of the aminopropanol group actually takes place in the very early stages of the amidation sequence. This possibility was clearly ruled out by (i) preliminary biochemical studies on the aminopropanolinserting enzyme system (3) and (ii) the fact that in such a case, mutants G624 and G626 complemented by the $\operatorname{cob} Q$ gene would be expected to accumulate cobinic acid $a, c-$ diamide instead of cobyrinic acid $a, c$-diamide (see above). Conversely, mutants G634 and G643, which are complemented by $c o b D$ and $c o b C$, respectively, and blocked in the aminopropanol-inserting reactions, have been shown to accumulate cobyric acid (8), as expected in the case of a late $(R)$-1-amino-2-propanol insertion.

From knowledge gained during studies of cobyrinic acid $a, c$-diamide synthase (11) and cobyric acid synthase, the sequence of amidations and adenosylation of the corrin macrocycle in $\boldsymbol{P}$. denitrificans in vivo appears to be much simpler than that suggested by Bernhauer et al. for $\boldsymbol{P}$. shermanii (2) for the following reasons. (i) Only two enzymes are required for the whole set of amidations, the first one carrying amidation at position $c$ followed by $a$ and the second one at positions $b, d, e$, and $g$. (ii) The four last amidations are carried out in a specific order (which was not determined). (iii) Insertion of the Ado group presumably takes place at the stage of cobyrinic acid $a, c$-diamide. Our 
study of $\boldsymbol{P}$. denitrificans cob(I)alamin adenosyltransferase fully confirmed this latter conclusion (10). The obligatory role of cobyric acid in cobalamin biosynthesis (Fig. 2) claimed by Friedrich is not rigorously proved yet but is strongly suggested, since no partially amidated corrinoids with the $(R)$-1-amino-2-propanol have been detected in $P$. denitrificans. Enzymatic studies on this (R)-1-amino-2-propanol-inserting enzyme system are now in progress.

\section{ACKNOWLEDGMENTS}

J. Lunel, P. E. Bost, and J.-C. Brunie are acknowledged for their support during this work. We also thank D. Faucher and F. Cuine for the $\mathrm{N}$-terminal sequencing of the protein.

This work was supported by the Rhône-Poulenc S.A. Health Division and by Rhône-Poulenc Rorer S.A.

\section{REFERENCES}

1. Bartosinski, B., B. Zagalak, and J. Pawelkiewicz. 1967. The route of vitamin $\mathrm{B}_{12}$ biosynthesis in Propionibacterium shermanii. Biochim. Biophys. Acta 136:581-584.

2. Bernhauer, K., F. Wagner, H. Michna, P. Rapp, and H. Vogelmann. 1968. Zur chemie und biochemie der corrinoide. XXIX. Biogenesewege von der cobyrinsäure zur cobyrsäure und zum cobinamid bei Propionibacterium shermanii. Hoppe-Seyler's Z. Physiol. Chem. 349:1297-1309.

3. Blanche, F., L. Debussche, A. Famechon, and D. Thibaut. Unpublished data.

4. Blanche, F., L. Debussche, D. Thibaut, J. Crouzet, and B. Cameron. 1989. Purification and characterization of $S$-adenosylL-methionine:uroporphyrinogen III methyltransferase from Pseudomonas denitrificans. J. Bacteriol. 171:4222-4231.

5. Blanche, F., D. Thibaut, M. Couder, and J.-C. Muller. 1990. Identification and quantitation of corrinoid precursors of cobalamin from Pseudomonas denitrificans by high-performance liquid chromatography. Anal. Biochem. 189:24-29.

6. Cameron, B., K. Briggs, S. Pridmore, G. Brefort, and J. Crouzet. 1989. Cloning and analysis of genes involved in coenzyme $\mathrm{B}_{12}$ biosynthesis in Pseudomonas denitrificans. J. Bacteriol. 171:547-557.

7. Casadaban, M. J., A. Martinez-Arias, S. T. Shapira, and J. Chou. 1983. $\beta$-Galactosidase gene fusion for analysing gene expression in Escherichia coli and yeast. Methods Enzymol. 100:293-308.

8. Crouzet, J., L. Cauchois, F. Blanche, L. Debussche, D. Thibaut, M.-C. Rouyez, S. Rigault, J.-F. Mayaux, and B. Cameron. 1990. Nucleotide sequence of a Pseudomonas denitrificans 5.4-kilobase DNA fragment containing five cob genes and identification of structural genes encoding $S$-adenosyl-L-methionine:uroporphyrinogen III methyltransferase and cobyrinic acid $a, c$-diamide synthase. J. Bacteriol. 172:5968-5979.

9. Crouzet, J., S. Lévy-Schil, B. Cameron, L. Cauchois, S. Rigault,
M.-C. Rouyez, F. Blanche, L. Debussche, and D. Thibaut. 1991. Nucleotide sequence and genetic analysis of a 13.1-kilobase-pair Pseudomonas denitrificans DNA fragment containing five $c o b$ genes and identification of structural genes encoding cob(I) alamin adenosyltransferase, cobyric acid synthase, and bifunctional cobinamide kinase-cobinamide phosphate guanylyltransferase. J. Bacteriol. 173:6074-6087.

10. Debussche, L., M. Couder, D. Thibaut, B. Cameron, J. Crouzet, and F. Blanche. 1991. Purification and partial characterization of cob(I)alamin adenosyltransferase from Pseudomonas denitrifcans. J. Bacteriol. 173:6300-6302.

11. Debussche, L., D. Thibaut, B. Cameron, J. Crouzet, and F. Blanche. 1990. Purification and characterization of cobyrinic acid a,c-diamide synthase from Pseudomonas denitrificans. J. Bacteriol. 172:6239-6244.

12. Ford, S. H. 1985. Amidation of, and (R)-1-amino-2-propanol attachment to, the corrin ring during vitamin $B_{12}$ biosynthesis by Clostridium tetanomorphum extracts. Biochim. Biophys. Acta 841:306-317.

13. Friedmann, H. C., and L. M. Cagen. 1970. Microbial biosynthesis of $\mathrm{B}_{12}$-like compounds. Annu. Rev. Microbiol. 24:159-208.

14. Friedrich, W. 1965. Zur biochemie der nucleotidfreien vitamin $B_{12}$-carbonsäuren. Biochem. Z. 342:143-160.

15. Friedrich, W., and W. Sandeck. 1965. Zur kenntnis der amidierung nucleotid- und isopropanolaminfreier $B_{12}$-polycarbonsäuren durch Propionibacterium shermanii. Z. Naturforsch. 20b:79-80.

16. Hayward, G. C., A. O. Hill, J. M. Pratt, N. J. Vanston, and R. J. P. Williams. 1965. The chemistry of vitamin $B_{12}$. IV. The thermodynamic trans-effect. J. Chem. Soc. 1965:6485-6493.

17. Hogenkamp, H. P. C. 1975 . The chemistry of cobalamins and related compounds, p. 21-73. In B. M. Babior (Ed.), Cobalamin. Biochemistry and pathophysiology. John Wiley \& Sons, Inc., New York.

18. Irion, E., and L. Ljungdahl. 1965. Isolation of factor $\mathrm{III}_{\mathrm{m}}$ and cobyric acid coenzyme plus other $\mathbf{B}_{12}$ factors from Clostridium thermoaceticum. Biochemistry 4:2780-2790.

19. Leong, L. S., G. S. Ditta, and D. R. Helinski. 1982. Identification of a cloned gene coding for $\delta$-aminolevulinic acid synthetase from Rhizobium melitoti. J. Biol. Chem. 257:8724-8730.

20. Rapp, P. 1973. Amidierung von corrinoidcarbonsäuren in rohextrakten aus Propionibacterium shermanii. Hoppe-Seyler's Z. Physiol. Chem. 354:136-140.

21. Webb, E. C. 1984. Recommendations of the nomenclature committee of the International Union of Biochemistry on the nomenclature and classification of enzyme-catalysed reactions. Academic Press, Inc., Orlando, Fla.

22. Weissbach, H., J. N. Ladd, B. E. Volcani, R. D. Smyth, and H. A. Barker. 1960. Structure of the adenylcobamide coenzyme: degradation by cyanide, acid, and light. J. Biol. Chem. 235: 1462-1473.

23. Zalkin, H. 1985. Glutamine amidotransferases. Methods Enzymol. 113:263-264. 